Revista Española de

I n v estig a ción

Cr i m i noló g i c a

\title{
La medición y evaluación de la criminalidad organizada en España: ¿Misión Imposible?
}

\author{
Andrea Giménez-Salinas Framis ${ }^{1}$ \\ Luis de la Corte Ibáñez \\ Laura Requena Espada \\ Manuel de Juan Espinosa \\ Universidad Autónoma de Madrid
}

Original recibido el 01 de noviembre de 2009

\section{RESUMEN}

Este artículo presenta un análisis exploratorio de las posibilidades de medición de la criminalidad organizada ${ }^{2}$ en España. Inicialmente, se abordan las dificultades de medición del crimen organizado referentes a su definición, la complejidad de su identidad, las fuentes de datos existentes y las posibilidades de obtención de los mismos. A continuación, se procede a la medición de la delincuencia organizada en España, en los últimos diez años, a partir de datos policiales. Dicha medición se plantea en base a tres dimensiones: las organizaciones criminales, las actividades o mercados de la delincuencia organizada y las actividades instrumentales necesarias para el desarrollo del crimen organizado.

Palabras clave: crimen organizado, medición crimen organizado, delincuencia organizada, organizaciones criminales.

\begin{abstract}
This article is an explorative analysis of the measurement of organized crime in Spain. The first part of the article explains the principal obstacles to measure organized crime, especially those regarding its definition, its identity, the existing sources of data and the access to organized crime data. The second part is a description of organized crime in Spain by means of official police data in the last ten years. The measurement explores three complementary dimensions: organized crime groups, activities organized crime markets and instrumental activities to develop organized crime.
\end{abstract}

Key words: organized crime, organize crime measurement, criminal organizations

\footnotetext{
${ }^{1}$ Correspondencia: andrea.gimenezsalinas@uam.es

${ }^{2}$ En este trabajo se utilizaran indistintamente los términos criminalidad organizada, crimen organizado y delincuencia organizada.
}

Revista Española de Investigación Criminológica

Artículo 9, Número 7 (2009) www.criminología.net

ISSN: $1696-9219$ 


\section{INTRODUCCIÓN}

La criminalidad organizada es un fenómeno de reciente interés político en nuestro país que coincide con una corriente internacional caracterizada por el consenso sobre la creciente amenaza de la criminalidad organizada en Europa (OCTA, 2007, 2008) ${ }^{3}$. Desde el punto de vista académico, el interés por la criminalidad organizada tuvo su punto de inflexión en los años 90 y estuvo protagonizado por el debate sobre su existencia y sobre su definición. En la actualidad, parece que podemos dar por superada la controversia conceptual y, siendo conscientes de las dificultades y limitaciones conceptuales del fenómeno, los esfuerzos se concentran en desarrollar nuevas formas de medición y evaluación de este fenómeno complejo y singular (Von Lampe 2004, Vander Beker 2004, Albanese 2000, 2008).

El debate sobre las formas de medición y evaluación constituye, sin duda, uno de los retos a los que nos enfrentamos en el conocimiento de la criminalidad organizada en nuestro país. La búsqueda de formas de medición más precisas, de métodos de evaluación más rigurosos y de modos de fomentar la transferencia de la información relacionada con dicha criminalidad, son cuestiones que debemos abordar en el caso español por dos motivos principales: 1) para poder contrastar empíricamente el consenso internacional en torno a la supuesta gravedad y ascenso imparable de la criminalidad organizada en nuestro país y en los países de nuestro entorno, y 2) para reaccionar frente a dicha criminalidad con estrategias adecuadas a las dimensiones y características locales, sin optar por reacciones extremas por sobredimensionar su magnitud y potencial peligrosidad.

A través de los informes oficiales disponibles, tanto nacionales como internacionales, es difícil hacerse una idea sobre los índices de crimen organizado de cada uno de los países ni cómo nos encontramos en referencia a los países vecinos. Los informes internacionales y europeos (OCTA -Organized Crime Threat Assessments) sobre criminalidad organizada incorporan información cualitativa y descriptiva respecto al crimen organizado, pero carecen de información cuantitativa para poder realizar evaluaciones comparativas entre los países. Otros

\footnotetext{
${ }^{3}$ Los informes abiertos que publica EUROPOL sobre delincuencia organizada en Europa coinciden en calificar la criminalidad organizada como un fenómeno creciente en Europa que amenaza con empeorar en el futuro. EUROPOL publica anualmente los EU Organized Crime Threat Assessments que, como su título bien indica, son informes de prospectiva que evalúan la amenaza de la delincuencia organizada en Europa. Dichos informes se elaboran a partir de los informes recibidos de los distintos pá́ses pero no incluyen estadísticas ni datos comparados sobre grupos delictivos o actividades de crimen organizado en Europa.
}

Revista Española de Investigación Criminológica

Artículo 9, Número 7 (2009) www.criminología.net

ISSN: 1696-9219 
organismos que ofrecen información estadística comparada sobre la criminalidad, tampoco aportan suficiente información sobre los delitos relacionados con la criminalidad organizada ${ }^{4}$.

El presente artículo ${ }^{5}$ tiene dos objetivos. El primero de ellos es exponer y analizar las dificultades de medición del crimen organizado, especialmente las barreras referidas al fenómeno y las dificultades directamente relacionadas con las fuentes de datos y la obtención de los mismos. El segundo objetivo es medir el crimen organizado en España de los últimos diez años, a partir de las posibilidades que nos ofrecen los datos sobre el crimen organizado procedentes de fuentes policiales. Este segundo objetivo se llevará a cabo a partir de mediciones referidas a tres dimensiones o facetas de este complejo fenómeno: las organizaciones criminales, sus actividades o mercados del crimen organizado y las actividades instrumentales necesarias para su desarrollo.

\section{2. ¿QUÉ QUEREMOS MEDIR CUANDO HABLAMOS DE CRIMINALIDAD ORGANIZADA?}

El primer aspecto que debemos abordar para evaluar un problema y proceder a su medición es determinar cuál es realmente el problema y en qué consiste. Por tanto, la definición del mismo se convierte en el elemento clave que debemos tratar en primer lugar para poder medir o evaluar un fenómeno.

Sobre la criminalidad organizada, se han escrito importantes contribuciones intentando llegar a un consenso sobre la existencia o no de un tipo especial de crimen denominado organizado, y sobre qué características debería tener dicho fenómeno. Las últimas décadas de la historia de la literatura sobre crimen organizado han estado marcadas por el debate sobre su definición y el cuestionamiento de su legitimidad.

Por un lado, los defensores de la teoría de la conspiración (alien conspirancy) han respaldado una idea del crimen organizado como categoría propia y distinta de otros fenómenos delictivos, y cuyo desarrollo conduce a una erosión sistemática de las bases económicas, políticas y sociales del Estado. Dicha postura, representada por el pionero en el estudio de la delincuencia organizada en Estados Unidos, Donald R. Cressey (1969), en su mítica obra The Theft of the Nation, pervive con fuerza en la actualidad y su argumentación ha llegado a la

${ }^{4}$ Los informes del European Sourcebook of Crime and Criminal Justice Statistics sólo ofrecen información relativa a tráfico de drogas y las encuestas de victimación internacionales (IVCS), no incorporan información sobre este tipo de delitos.

${ }^{5}$ La elaboración de este trabajo no hubiera sido posible sin la inestimable colaboración del Gabinete de Estudios para la Seguridad Interior del Ministerio del Interior (GESI). El GESI nos ha facilitado tanto datos de delincuencia organizada procedentes de los informes que se realizan sobre la materia como asesoramiento e información relativa a la metodología utilizada para recogerlos. Agradecemos especialmente su colaboración aunque las afirmaciones y valoraciones que se incluyen en el artículo son responsabilidad de los autores del mismo.

Revista Española de Investigación Criminológica

Artículo 9, Número 7 (2009) www.criminología.net

ISSN: $1696-9219$ 
Europa actual calando hondo en las instancias políticas que persiguen el control de esta forma de delincuencia.

Otra postura, completamente opuesta, cuestiona la existencia de la delincuencia organizada como una categoría distinta de delito, por el mero hecho de ser organizada, y considera que únicamente el interés político, movido por un afán de encontrar amenazas o enemigos, justifica dicha construcción categorial. Desde este punto de vista, se considera que las actividades que desarrollan los grupos criminales ya se encuentran criminalizadas y, por tanto, su castigo es suficiente (Levy, 2003, Sánchez García de Paz, 2005, Paoli, 2002). Por tanto, el castigo de la asociación criminal y la necesidad de crear medios extraordinarios de investigación para este tipo de delitos, obedecería a una voluntad política tendente a justificar una mayor represión y persecución donde predomina la finalidad proactiva y procedimental.

Desde el punto de vista criminológico, se considera que este debate conceptual ha quedado hoy relegado a un segundo plano, habiendo llegado al consenso de considerar la criminalidad organizada como un fenómeno con identidad propia (Abadinsky, 2003, Albanese, 2000, Morselli, 1997). Así, el crimen organizado aparece caracterizado por una suma de factores que dan forma a un fenómeno complejo y necesitado de un abordaje teórico y operativo propio.

\begin{tabular}{|l|l|}
\hline \multirow{4}{*}{ Indicadores obligatorios } & Colaboración de dos o más personas \\
\cline { 2 - 2 } & Búsqueda de beneficios de poder \\
\cline { 2 - 2 } & Permanencia en el tiempo \\
\cline { 2 - 2 } & Sospecha de comisión de delitos graves \\
\hline \multirow{5}{*}{ Mínimo tres } & Reparto de tareas específicas \\
\cline { 2 - 2 } & Mecanismo de control y de disciplina interna \\
\cline { 2 - 2 } & Actividad Internacional \\
\hline & Empleo de violencia e intimidación \\
\hline & Empleo de estructuras comerciales y económicas \\
\hline & Implicación en el blanqueo de capitales \\
\cline { 2 - 2 } & Influencias políticas, medios de comunicación, etc. \\
\hline
\end{tabular}

Tabla 1. Indicadores de Criminalidad organizada utilizados por Europol

A pesar de la existencia de ciertas discrepancias respecto a los factores que identifican el crimen organizado, existen características compartidas por la mayoría de autores. La tabla 1 recoge las características definitorias utilizadas por EUROPOL para clasificar los grupos de delincuencia organizada desde un punto de vista operativo. Los indicadores utilizados recogen en gran medida las características definitorias que mayor consenso tienen en la literatura académica y son el fruto de un largo trabajo realizado desde EUROPOL a favor de la lucha 
contra la delincuencia organizada ${ }^{6}$. Además, dichos indicadores coinciden con los aspectos esenciales de la criminalidad organizada resaltados por las definiciones internacionales: la existencia de a) una organización, b) una estructura, c) una finalidad común, y d) la comisión de delitos graves.

\subsection{Una medida para un concepto multidimensional}

Íntimamente relacionadas con la definición de un fenómeno, se encuentran las características que lo identifican y lo distinguen de otros fenómenos.

Si lo que queremos es medir la criminalidad organizada, lo primero que nos encontramos cuando analizamos su contenido, es que se trata de un fenómeno con múltiples dimensiones interrelacionadas entre sí, que deben tomarse en cuenta para su medición y evaluación. Consideraremos tres dimensiones esenciales para medir dicha criminalidad y para evaluar sus características: grupos, mercados y actividades instrumentales. La primera debe integrar a los grupos u organizaciones de crimen organizado. La segunda dimensión se refiere a las actividades o mercados legales o ilegales que son objetivo del crimen organizado y que constituyen la actividad lucrativa principal del grupo. La tercera y última está representada por aquellas actividades que constituyen los medios de desarrollo del crimen organizado, actividades que son necesarias para la supervivencia de los grupos de crimen organizado y que se consideran actividades inseparables a la principal actividad delictiva de los mismos. Estos tres elementos: grupos, mercados y actividades instrumentales, están presentes en la criminalidad organizada de forma interrelacionada y juntos constituyen su identidad compleja.

\footnotetext{
${ }^{6}$ Europol lleva años elaborando una metodología común de recogida de datos sobre delincuencia organizada a desarrollar por cada uno de los países que participan en la elaboración de los informes que publica EUROPOL. Así, el OCTA es fruto de la aplicación estandarizada de una metodología concreta de recogida de datos en los distintos países. Fruto del trabajo continuado y la experiencia acumulada son los indicadores comunes que definen y clasifican los grupos de delincuencia organizada.
}

Revista Española de Investigación Criminológica

Artículo 9, Número 7 (2009) www.criminología.net

ISSN: $1696-9219$ 


\section{Grafico 1. Dimensiones del crimen organizado}

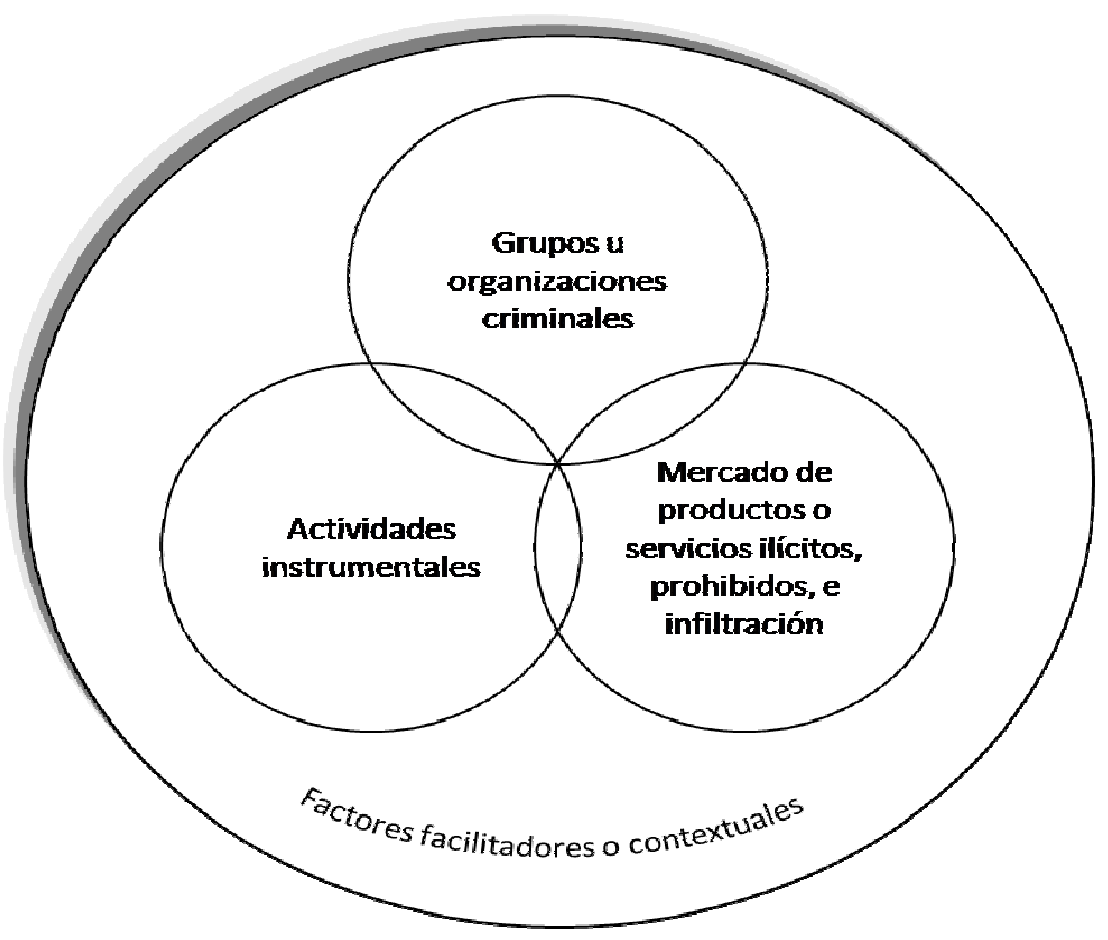

Como puede apreciarse en la Gráfica 1, para poder evaluar o medir la delincuencia organizada existente en un determinado lugar, no sólo tendremos que conocer el tipo y las características del grupo al que nos enfrentamos, también los mercados a los que se dedica principalmente, y las actividades que desarrolla para su conducción en clandestinidad, para la defensa del mercado ilegal y el desarrollo del mismo, etc. Dichas dimensiones incorporan indicadores especialmente relevantes para conocer de forma integral, la capacidad, los recursos, la potencialidad de dicho grupo, su actividad y su capacidad de penetración social, económica e institucional. Dichas dimensiones deber considerarse conjuntamente para medir la delincuencia organizada en un determinado lugar pero, en especial, para evaluar las características y potencialidad de los grupos criminales.

Finalmente, las actividades de delincuencia organizada no se desarrollan en el vacío. Normalmente las acciones de un grupo de crimen organizado se desarrollan en un contexto específico porque existen determinadas condiciones que facilitan el surgimiento de un fenómeno de este tipo. Es decir, dichas condiciones facilitadoras constituyen el caldo de cultivo para que se genere un determinado mercado ilegal que pueda proporcionar oportunidades a los grupos criminales existentes y potenciar la evolución ascendente de la criminalidad organizada en un determinado lugar. Factores económicos, políticos, y sociales han sido también identificados y utilizados como indicadores para la evaluación del riesgo de emergencia de 
criminalidad organizada en un determinado lugar (Albanese, 2001, 2008, Vander Beken, 2004, Williams y Godson, 2002).

\section{3. ¿LA DELINCUENCIA ORGANIZADA SE MIDE COMO CUALQUIER OTRO DELITO?}

Como la medida de un determinado concepto está íntimamente ligada a la naturaleza del problema que queremos medir y de su composición, cuanto mayor es la complejidad del problema, mayor será la complejidad de la medida.

Respecto a los fenómenos criminales en general, hay delitos que corresponden a conductas individuales que son relativamente fáciles de medir. Los homicidios, robos, agresiones sexuales, coacciones, amenazas, etc., suelen denominarse delitos convencionales, y consisten en conductas individuales asociadas a un individuo en particular (Van Dijk, 2004 y 2008). En estos casos, un acto constituye un hecho cuantificable aisladamente y son hechos sobre los que suele existir un consenso en su definición internacionalmente compartida. Para medir dichos delitos convencionales, conocemos los instrumentos de medida que nos permiten hallar índices de criminalidad, más o menos adecuados a su realidad delincuencial, según la fuente de datos por la que optemos ${ }^{7}$.

Sin embargo ¿Qué ocurre con los delitos complejos o no convencionales, como el crimen organizado? (Buscaglia y Van Dijk, 2003), ¿Son delitos parecidos a los convencionales? ¿Equivalentes?, ¿Podemos utilizar los mismos instrumentos de medida?, ¿Qué problemas nos encontramos para medir su magnitud y conocer sus dimensiones? Como es lógico, dichos delitos no convencionales son considerados como una categoría separada y, por lo tanto, obedecen a factores y causas distintas que los delitos convencionales (Farrington, 2005, Kleemans \& De Poot, 2008). En este sentido, teóricamente podríamos tener países con índices elevados en delitos convencionales como los robos, las agresiones sexuales, etc., y tener índices no tan elevados en delitos no convencionales, como la delincuencia organizada (Van Dijk y Nevala: 2002).

Sin embargo, decir que ambos tipos de delitos son distintos no significa que no estén relacionados entre sí. Es más, algunos autores apuntan a que, cuando se obtienen índices

\footnotetext{
${ }^{7}$ Disponemos de las estadísticas judiciales y policiales que nos ayudan a conocer la realidad delincuencial conocida por los agentes de control y por los juzgados que no la realidad delincuencial de un determinado país. Así, para poder conocer la cifra negra o los delitos que no llegan a conocerse por parte de la policía se idearon, por un lado, las encuestas de victimización que nos proporcionan información sobre los delitos sufridos por la población general y sobre la confianza de la población en las instancias de control y, por otro, las encuentras de autoinforme que proporcionan valiosa información sobre la pequeña delincuencia especialmente en población juvenil (Garrido, Stangeland y Redondo, 2006).
} 
elevados de delincuencia convencional y a la vez índices bajos en delincuencia organizada registrados por la policía, se puede predecir una mayor presencia de delincuencia organizada en la realidad. Es decir, tal y como apunta Van Dijk (2008), es de esperar que el número de casos registrados por la policía en relación al crimen organizado, correlacione inversamente con la seriedad del problema en un determinado país. Esta afirmación tiene una explicación relativamente sencilla. Dos de los factores facilitadores más importantes de delincuencia organizada en un determinado país son: 1) la ausencia de mecanismos de control específicos para la lucha contra la criminalidad organizada y 2) los elevados niveles de corrupción (Albanese, 2001). Si en un lugar determinado confluyen dichos factores, lo lógico será tener índices bajos de delincuencia organizada registrados provocados por el escaso nivel de detección policial.

Además de los problemas mencionados, los fenómenos no convencionales o complejos como la delincuencia organizada, se caracterizan por una serie de particularidades que dificultan su medición y evaluación a través de fuentes oficiales y no oficiales.

En primer lugar, el crimen organizado requiere esencialmente la participación de varias personas formando un grupo o una organización. Este hecho supone una importante limitación a la hora evaluar su volumen ya que, la unidad de medida pasa del sujeto al grupo. El grupo, como organización, puede ser de muchos tipos y presentar diversas características. Así pues, medir el número de personas detenidas y/o condenadas por crimen organizado, puede ser el primer paso que nos acerque a conocer la cantidad de delitos cometidos por esta actividad, sin embargo, nada nos dice sobre los grupos y su composición.

Un segundo paso consiste en conocer el número de grupos detectados. Sin embargo, medir el volumen de crimen organizado de un país sólo por el número de grupos criminales registrados en un determinado año, no es una medida muy precisa por dos motivos: 1) Al no contemplar la diversidad de composición de cada grupo $(3,6, . . \mathrm{N}$ miembros) estamos otorgando la misma importancia a todos los grupos conocidos y 2) el número de grupos no nos proporciona la información necesaria para que podamos valorar características de los grupos que son relevantes para su evaluación: nivel de estructura y organización, nivel de penetración económica y política, división de tareas entre los miembros, infraestructura y recursos, etc.

En segundo lugar, un grupo de criminalidad organizada puede desarrollar manifestaciones delictivas diversas, no todas igual de importantes a la hora de evaluar la potencialidad del grupo y su nivel de consolidación. No debemos conceder la misma importancia a las actividades que constituyen el negocio o mercado ilícito propio del grupo, o las actividades ilegales que el grupo realiza para mantener su supervivencia. De esta distinción y la importancia otorgada a las actividades principales e instrumentales, hablaremos más adelante con detalle. 
En último lugar, la transnacionalidad del fenómeno también dificulta enormemente su medición local. Si un grupo actúa en diversos países a la vez, como ocurre frecuentemente en la actualidad, el hecho que identifiquemos una ramificación en un determinado lugar nos aportará información sobre el volumen correspondiente a una parte del grupo criminal pero no respecto a la totalidad del mismo.

\section{PARA MEDIR LA CRIMINALIDAD ORGANIZADA ¿POR DÓNDE EMPEZAMOS?}

Hemos comentado la dificultad que entraña la medición de la delincuencia organizada relativa a dos obstáculos principales: la multidimensionalidad del concepto y su identidad grupal. Pero, ¿qué posibilidades presenta la aplicación de instrumentos de medición convencionales y alternativos utilizados para otros delitos? Los instrumentos más comúnmente utilizados para medir el delito convencional son los cuestionarios de autoinforme y los de victimización. Si bien estos instrumentos se han mostrado útiles para la medición del delito convencional, su empleo en el ámbito de delitos complejos, como el crimen organizado, plantea serios problemas (Medina Ariza, 1999).

En primer lugar, el autoinforme consiste en una encuesta (anónima y de libre participación), orientada a autores o potenciales autores, sobre su comisión de conductas ilegales. Al orientarse sobre los autores, la utilidad potencial de este tipo de instrumentos para medir el crimen organizado es claramente prometedora. No obstante, su debilidad estriba tanto en la colaboración de los participantes, como en la fiabilidad de sus respuestas. En cuanto al primer punto, la coacción y amenazas que una organización ejerce sobre sus miembros dificultarían seriamente su colaboración en este tipo de encuestas. En cuanto al segundo aspecto, las encuestas de autoinforme se dirigen usualmente a población juvenil y sus preguntas tienden a circunscribirse a delitos convencionales, de menor gravedad. Esto supone una diferencia con respecto a la delincuencia organizada, caracterizada por la implicación mayoritaria de delincuentes adultos y la comisión de delitos graves (Kleemans \& De Poot, 2008). Es más, preguntar sobre la conducta ilegal de un autor en el campo de la delincuencia organizada no sólamente tendría que abordar delitos graves sino que dichos delitos tendrían que ser enmarcados en el conjunto de acciones de una organización, mediante preguntas sobre la misma que garantizaran que dichas actividades delictivas no pertenecen al ámbito particular, sino que son el resultando de una actividad orquestada y organizada. Lógicamente, todo ello comprometería seriamente la fiabilidad de las respuestas.

Una forma alternativa más eficaz de medición seria recurrir a encuestas de victimización. Este tipo de instrumentos se han utilizado para medir la delincuencia organizada, empleando muestras restringidas de hombres de negocios o de sectores comerciales concretos 
que son las poblaciones mayormente victimizadas por la extorsión y la corrupción. Por ejemplo, en Italia se llevan a cabo este tipo de encuestas por parte de asociaciones empresariales. En una de las encuestas llevadas a cabo en Calabria en 1998, se reveló que el 70\% de las tiendas de esta localidad sufrían extorsiones y 4 de 5 también las sufrían en Palermo y Catania (Jamieson, 2000).

Utilizando la misma metodología del International Criminial Victimization Survey (ICVS) se elaboró una encuesta de victimización denominada ICBS (International Criminal Bussines Survey) que en 1995 se aplicó a muestras de ejecutivos en 10 países desarrollados (Van Dijk y Terlouw, 1996). En estas encuestas se les preguntaba a los entrevistados sobre si habían sido víctimas de robos o corrupción, y sobre su percepción sobre la extorsión y las demandas de protección a cambio de dinero existentes en su sector. En la mayoría de países de Europa occidental, entre 7 y $15 \%$ de los entrevistados encontraron que estas prácticas eran muy comunes o bastante comunes. Las tasas más altas se encontraron en la República Checa y Hungría. Algo más tarde, en el 2000, se utilizó una versión del cuestionario ICBS, con preguntas sobre crimen organizado y corrupción, en una encuesta UNICRI- Gallup executed survey, entre las capitales de nueve países en Europa Central y del Este. Alrededor de un 15\% de los encuestados consideraron que la intimidación y la extorsión era una práctica común o bastante común en su sector.

También se han realizado encuestas sobre percepción del crimen organizado con poblaciones concretas. En esta línea, el World Economic Forum en su Global Competitiveness Report de carácter anual incorporó a partir de 2003 una encuesta a personas del mundo de los negocios, en la que una de las preguntas estaba relacionada con la percepción de crimen organizado.

Los resultados de los ICBS sobre victimización de personas del mundo de los negocios con respecto al crimen organizado, y los estudios exploratorios sobre precepción del mismo, reflejan el potencial de este tipo de encuestas respecto a la recogida de información sobre el volumen y extensión de la delincuencia organizada y delitos complejos. Así pues, las encuestas de victimización aparecen como el tipo de instrumento preferido y preferible para una primera aproximación al problema de la medición del crimen organizado. Sin embargo, no siempre podemos acudir a este tipo de instrumentos y no siempre tenemos la posibilidad de realizar este tipo de estudios con una periodicidad suficiente para poder proceder a evaluaciones tanto puntuales como longitudinales. Pues bien, a falta de este tipo de encuestas en nuestro país, para conseguir nuestros objetivos tendremos que depender de los datos oficiales, con las limitaciones que dichos datos tienen para la delincuencia en general y para este tipo de delitos en particular. 


\section{CON LOS DATOS POLICIALES ¿QUÉ PODEMOS MEDIR Y EVALUAR?}

Centrándonos en los datos que recoge la policía para medir y evaluar la delincuencia organizada ¿es posible llegar a este objetivo con dichos datos? Nuevamente nos encontramos con serias dificultades. Para proceder, en primer lugar, nos deberíamos preguntar si existen datos sobre delincuencia organizada y de qué tipo son.

Para responder a esta pregunta, nos encontramos con un problema de fondo. En nuestro país no existe un delito como tal de delincuencia organizada. Existe el delito de asociación ilícita del art 515.1 del Código Penal, y también existen numerosos delitos referentes al tipo de actividades que desarrollan los supuestos grupos de criminalidad organizada.

En la base de datos estadística de seguridad que gestiona el Ministerio del Interior, resulta difícil obtener datos sobre el delito de asociación ilícita y los datos que se encuentran recogidos no se refieren únicamente a la delincuencia organizada. Por lo tanto, en un principio, deberíamos acudir a los distintos delitos cometidos por los sujetos implicados en la criminalidad organizada. Esto sería posible si se cumplieran dos condiciones: 1) que dicha base de datos sobre delincuencia pudiera desagregar todas las actividades que suelen estar relacionadas directa o indirectamente con la delincuencia organizada y, 2) que los datos estadísticos nos permitieran discriminar, de entre los delitos relacionados con la delincuencia organizada, aquellos que hubieran sido cometidos por miembros concretos de un determinado grupo perteneciente a la misma. En el caso español, lamentablemente no se dan ninguna de las dos condiciones. Por lo tanto, medir de forma precisa la delincuencia organizada registrada a partir de la base estadística de seguridad del Ministerio del Interior, es inviable hoy por hoy. En un futuro próximo, sí será posible gracias a los cambios que están introduciendo en las bases de datos en los dos últimos años ${ }^{8}$.

\section{LA DELINCUENCIA ORGANIZADA EN ESPAÑA A TRAVES DE LOS DATOS POLICIALES ${ }^{9}$}

Como ya avanzamos, el crimen organizado puede analizarse desde tres dimensiones: 1) grupos y sujetos, 2) mercados, y 3) actividades instrumentales o necesarias para el desarrollo de la criminalidad organizada. Teniendo en cuenta las limitaciones con las que contamos,

\footnotetext{
${ }^{8}$ En el año 2008 se ha producido una reforma importante de la plantilla de recogida de datos que tradicionalmente se utilizaba desde el Ministerio del Interior. En los años venideros tendremos mucha más información y mejoras sustanciales en la recogida de la información. Dentro de los cambios previstos se introduce un campo en el que se podrá desagregar del conjunto de delitos, los que se producen en el entorno de una organización criminal y pertenecen a la categoría genérica de delincuencia organizada.

${ }^{9}$ Los datos recogidos en dicho apartado han sido proporcionados por el Ministerio del Interior a partir de información recogida en los informes anuales que ser realizan sobre delincuencia organizada.
} 
presentaremos a continuación los datos obtenidos sobre estas dimensiones gracias a la colaboración del Ministerio del Interior a través del Gabinete de Estudios de Seguridad Interior. Nuestra finalidad con ello es doble, a) conocer la incidencia de la delincuencia organizada en los últimos diez años y, b) comprobar cómo será la instantánea que obtengamos de la evolución de la delincuencia organizada en la última década, en función de los datos que tomemos.

\subsection{Grupos y sujetos implicados en la delincuencia organizada}

En los últimos años se han realizado contribuciones relevantes desde la perspectiva del estudio de las organizaciones. De especial interés son aquellas que provienen de las teorías de redes sociales aplicadas a las organizaciones criminales (Mcillwain, 1999, Morselli, 2003) cuyo enfoque supone un paso adelante en el conocimiento de cómo los controles externos e internos a las mismas influyen en su forma y contenido. Si abordamos la medida y evaluación del crimen organizado desde un enfoque basado en la organización y estructura de los grupos, lo que obtendremos serán datos sobre el número de miembros que forman el grupo, estructura del grupo, etc. (Beare, 2000, Galeotti, 1998); forma y composición de las redes y distribución de poderes entre los miembros de dichas redes e intermediarios claves de las mismas (Morselli, 2009).

\section{El grupo como indicador}

La criminalidad organizada tiene como característica definitoria la existencia de una organización que realiza directamente las actividades ilegales. Desde esta perspectiva, la unidad de referencia para medir el crimen organizado será el grupo que configura una organización. Por tanto, una primera aproximación a la evaluación de la delincuencia organizada en España consiste en conocer el número de grupos que, desde la definición de delincuencia organizada, operan en nuestro país.

El gráfico $\mathrm{n}^{\mathrm{0}} 2$ nos muestra un histórico del número de grupos detectados por las fuerzas policiales entre 1998 y 2006. El cambio de color de las barras del histograma corresponde al cambio de criterio en la composición de los grupos de delincuencia organizada. Hasta el año 2001, se tomaba como grupo aquellos compuestos por 10 o más miembros. A partir de este año, también se consideró como grupo aquellos compuestos por 3 o más miembros. Pues bien, como puede observarse, existe un incremento de los grupos detectados del año 1998 al 2000, con un repunte especialmente importante en 2002 y luego una disminución en los años posteriores. 
Gráfico 2. Evolución de los grupos de delincuencia organizada entre 1998 y 2006*10

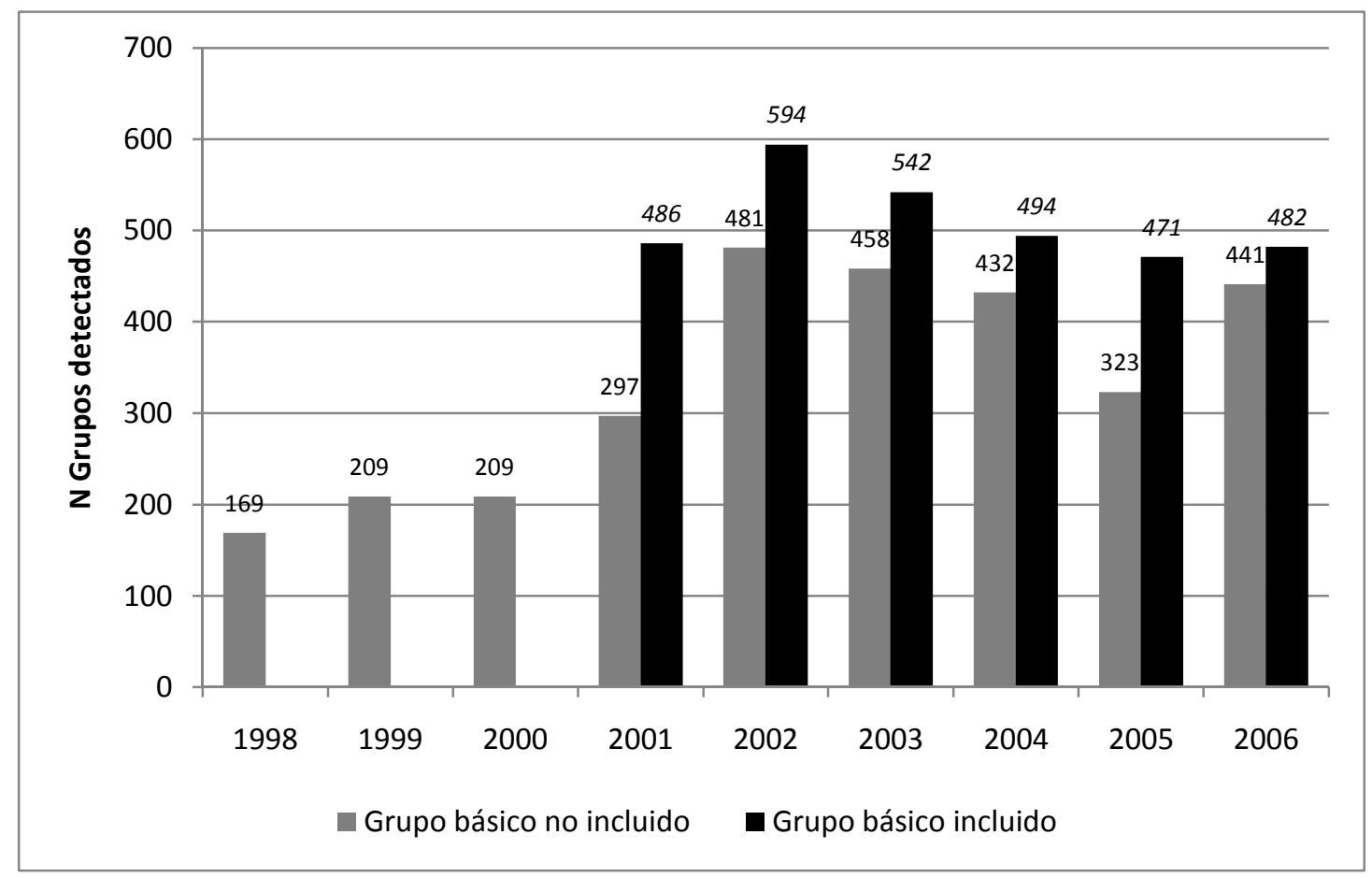

${ }^{*}$ Elaborada a partir de los datos del Ministerio del Interior.

Aunque estos datos son importantes, tienen serias limitaciones cuando se pretende una evaluación de la criminalidad organizada utilizando estos datos como único indicador de la misma. Como ya comentamos, considerar como medida el número de grupos supone incluir dentro de la categoría unidades equivalentes. En el caso de los grupos de criminalidad organizada, en ningún momento las unidades son equivalentes. El número de grupos, como criterio único, no nos informa sobre la extensión media de los grupos, su tipología, peligrosidad, nivel de penetración local, capacidades, recursos, por nombrar sólo algunos aspectos relevantes. Dicha limitación también impide estudios comparativos que nos proporcionen información relativa a nuestra posición respecto a otros países.

Los grupos de crimen organizado, para ser calificados como tales, tienen que cumplir una serie de indicadores que se han elaborado por consenso, como forma de clasificación (ver tabla 1). Es cierto que, conociendo el número de grupos registrados por las fuerzas policiales, podemos saber cuántos grupos cumplen los indicadores exigibles para entrar en la categoría de crimen organizado ya que, de no ser así, no habrían sido contabilizados. Sin embargo, al margen

\footnotetext{
${ }^{10}$ En el año 2003 se crea una nueva categoría de grupo básico, añadida a las tres categorías existentes anteriormente. También a partir de 2003 cambian los criterios de clasificación de los grupos eliminándose en número de integrantes como principal criterio.
}

Revista Española de Investigación Criminológica 
de estos datos quedan aspectos tan relevantes como son los indicadores específicos que cumplen cada grupo, y la cantidad de estos que los caracteriza ${ }^{11}$; una vía básica para su distinción y análisis. Además de éstas características, se sumarían un conjunto de variables que, en nuestra opinión, consideramos imprescindibles para la evaluación y medición de la criminalidad organizada (ver tabla 2)

\begin{tabular}{|l|}
\hline \multicolumn{1}{|c|}{ Variables } \\
\hline Extensión del grupo organizado \\
\hline Tipo de estructura organizativa \\
\hline Extensión territorial \\
\hline Actividad principal del grupo \\
\hline Existencia de más actividades principales \\
\hline Asociaciones con otros grupos y tipos de asociación \\
\hline Antigüedad conocida \\
\hline Nivel de sofisticación del grupo \\
\hline Nivel de influencia y corrupción \\
\hline Nivel de permeabilidad del grupo \\
\hline Nivel de infiltración económica: blanqueo de capitales \\
\hline Nivel de alianzas con empresas legales \\
\hline Nivel de violencia hacia el interior del grupo \\
\hline Nivel de violencia hacia el exterior del grupo \\
\hline
\end{tabular}

Tabla 2. Variables que pueden utilizarse para evaluar los grupos criminales

\section{Miembros de los grupos}

Una forma de aproximarnos a la extensión de los grupos para conocer su medida interna es considerar los miembros de los mismos.

Como el número de grupos existentes ya hemos visto que no nos permite comparar año a año la evolución de la delincuencia organizada ya que no incluimos dentro de la categoría unidades equivalentes, incluimos tanto el número de personas implicadas en el crimen organizado como la media de miembros por grupo para conocer si la evolución durante los últimos años es parecida.

El gráfico 3 muestra los datos sobre la extensión de los grupos (barras) incluyendo la media de miembros por grupo (líneas), como único dato existente referente a volumen interior del grupo. El cambio de color obedece al cambio de criterio de inclusión comentado en el gráfico anterior. Como puede observarse, en los últimos años (a excepción del 2008) el número

\footnotetext{
${ }^{11}$ Los indicadores se toman en consideración globalmente sin especificar qué indicador cumple cada uno de los grupos. Sería deseable discriminar esta información y otorgar pesos distintos a los indicadores establecidos para clasificar la delincuencia organizada, ya que no todos tienen la misma importancia a la hora de clasificar a los grupos.
}

Revista Española de Investigación Criminológica 
de personas implicadas en el crimen organizado desciende ligeramente. Sin embargo, la evolución de la media de miembros por grupo nos indica que éstos son cada vez menos numerosos.

Gráfico 3. Estimación del número de miembros 1998-2006*

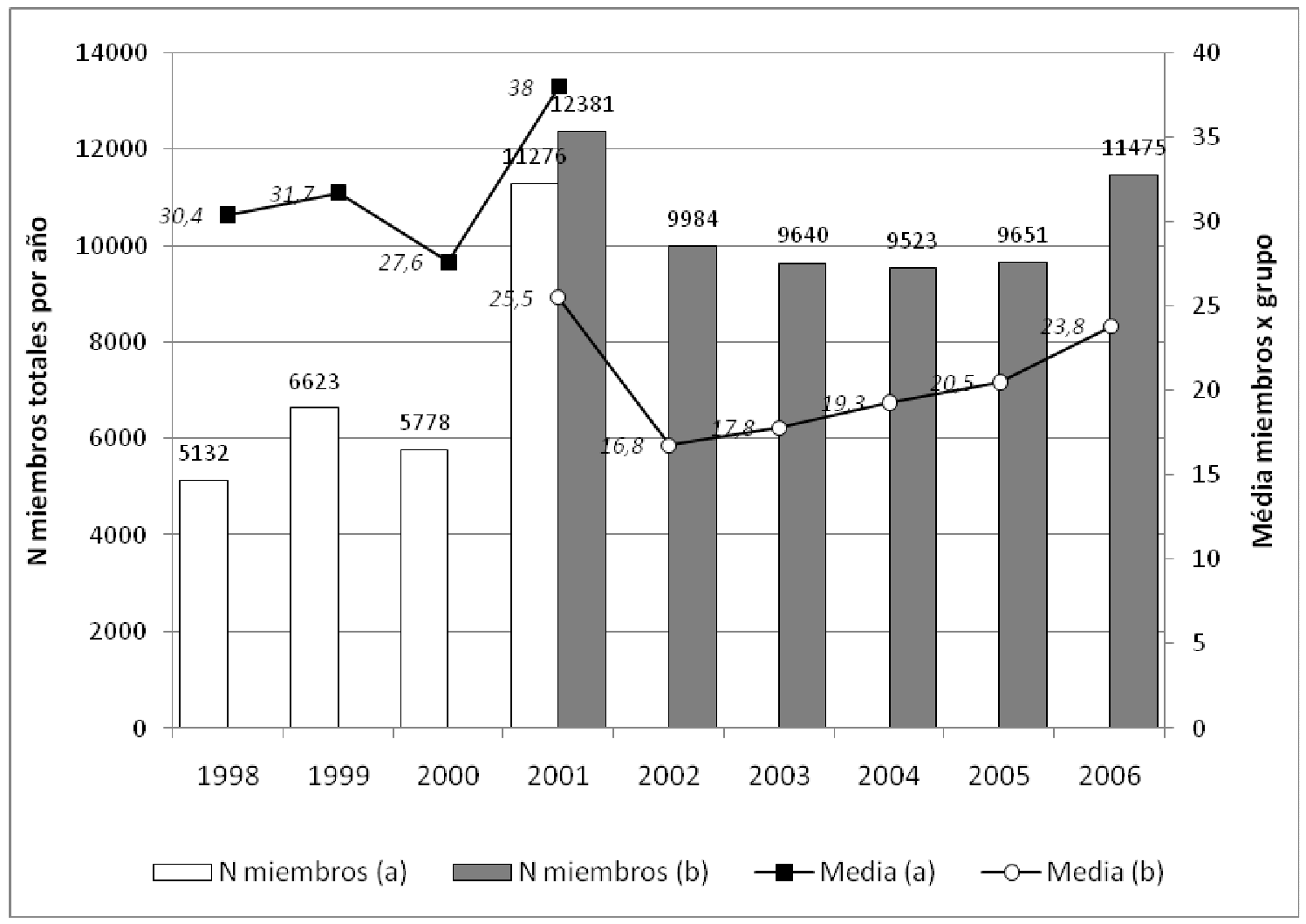

"Fuente: elaboración a partir de datos procedentes del Ministerio del Interior.

Este gráfico 3 nos describe una situación curiosa y nos aporta mayor información que el anterior. Parecería por los datos que los grupos detectados en los últimos años son cada vez más pequeños. Esto ¿quiere decir que estamos ampliando el concepto de crimen organizado para abarcar más grupos que antes no entraban en dicha categoría? Puede también querer decir que tenemos una tendencia al alza en delitos comunes ejecutados en grupo y con características que se acercarían a las propias del crimen organizado. Son hipótesis que deberíamos comprobar en el futuro a partir de una evaluación más precisa de los grupos. No obstante, para ello necesitaríamos más información relativa a las características de dichos grupos. 
Detenidos por delitos relacionados con el crimen organizado

Hemos visto como se describe la evolución de la criminalidad organizada en función de los datos sobre grupos con los problemas y limites mencionados. Más preciso es medir la delincuencia organizada a partir del número de detenidos por delincuencia organizada. Veamos si la tasa de detenidos por delincuencia organizada por 100.000 habitantes nos refleja otro panorama.

\section{Gráfico 4. Tasa de detenidos por delincuencia organizada por 100.000 habitantes*}

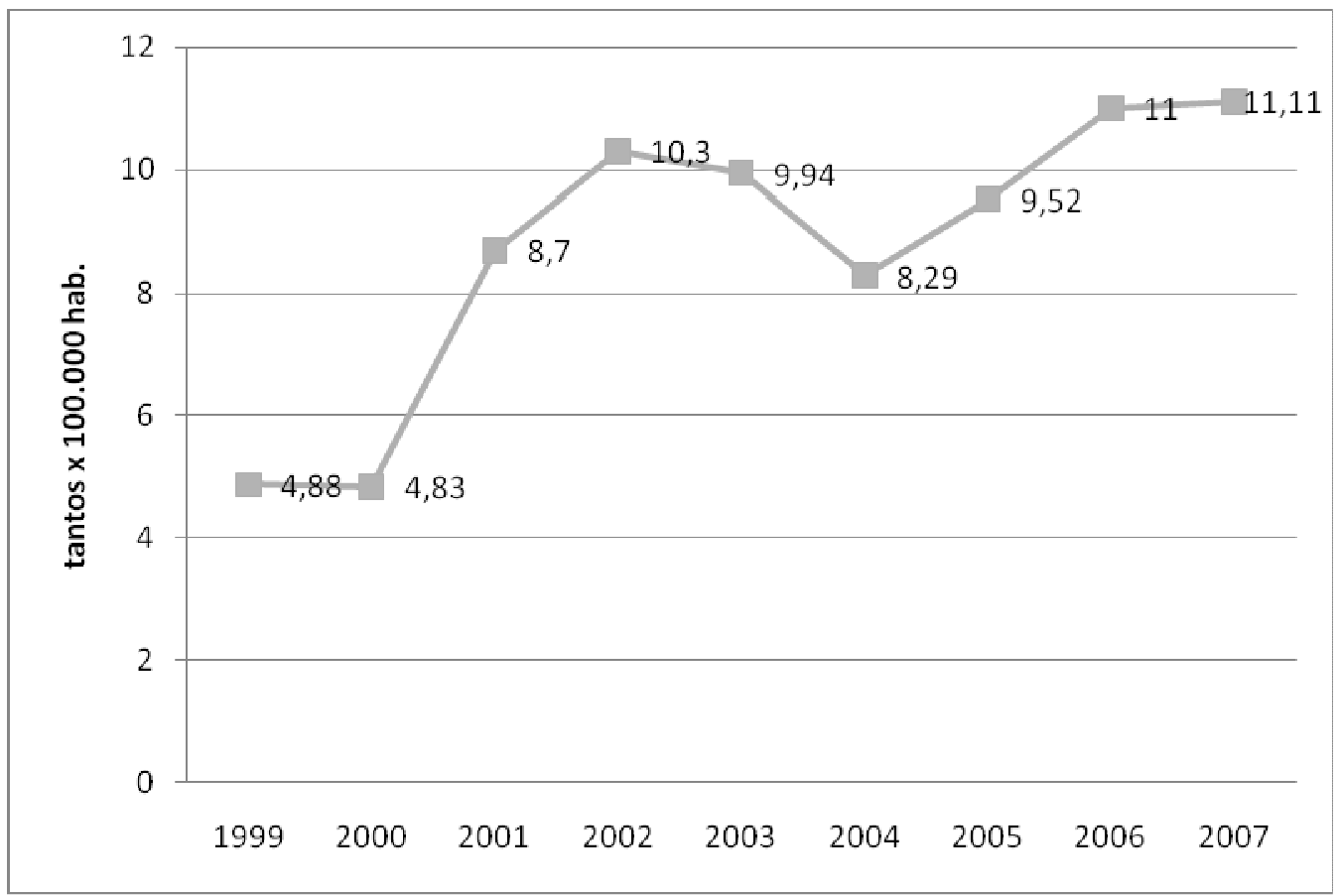

*Fuente: elaborada a partir de datos procedentes del Ministerio del Interior.

El grafico 4 nos muestra una evolución ascendente de la tasa de detenidos por 100.000 habitantes desde el año 2000 hasta el 2007, salvo el año 2003 y 2004 donde se produce un descenso. Esto quiere decir que las detenciones en materia de delincuencia organizada han aumentado progresivamente en los últimos diez años de forma casi continuada. Este aumento de las detenciones puede estar motivado por, al menos, dos causas. La primera de ellas pude estar relacionada con un aumento de la represión policial respecto a este problema y del aumento de recursos especializados creados para la represión del crimen organizado en los últimos años. Tanto el Cuerpo Nacional de Policía como la Guardia Civil tienen recursos especializados para la lucha contra el crimen organizado (UCO y UDYCO) y en 2006 se creó el Centro de 
Inteligencia contra el Crimen Organizado para la coordinación de operaciones e información en materia de investigación policial sobre el crimen organizado. La segunda de las causas puede estar relacionada con un aumento real de este tipo de delincuencia. Como, por el momento carecemos de datos que permitan contrastar la segunda de las afirmaciones, nos quedaremos con la primera de ellas.

\subsection{Actividades o mercados del crimen organizado}

En la literatura sobre delincuencia organizada se distingue entre varios tipos de actividades o mercados desarrollados por el crimen organizado. Unas son actividades principales y otras instrumentales o necesarias para el funcionamiento de los grupos de delincuencia organizada (Abadinsky, 2007). Las actividades principales o básicas del grupo son aquellos mercados ilegales a través de los cuales el grupo obtiene el principal beneficio económico. Las actividades instrumentales son, por el contrario, actividades necesarias para el desarrollo, mantenimiento y supervivencia de la organización y su negocio. Igualmente, dentro de las actividades principales, nos encontramos tres tipos de actividades principales relacionadas con el crimen organizado: a) la provisión de servicios ilícitos; b) la provisión de bienes ilegales y c) la infiltración de empresas legales (ver Tabla 3).

\begin{tabular}{|c|c|c|}
\hline \multirow{3}{*}{ Actividades principales } & Trafico de bienes ilícitos & $\begin{array}{l}\text { Tráfico de drogas } \\
\text { Tráfico de vehículos robados } \\
\text { Tráfico de armas } \\
\text { Falsificaciones moneda, marcas, medios de } \\
\text { pago } \\
\text { Contrabando productos }\end{array}$ \\
\hline & Trafico de servicios ilícitos & $\begin{array}{l}\text { Trata con fines explotación sexual } \\
\text { Tráfico inmigrantes } \\
\text { Fraudes y estafas organizadas }\end{array}$ \\
\hline & Infiltración economía legal & Blanqueo de capitales \\
\hline \multirow{4}{*}{$\begin{array}{c}\text { Actividades } \\
\text { instrumentales más } \\
\text { usuales }\end{array}$} & Violencia instrumental & $\begin{array}{l}\text { Coacciones, extorsiones, amenazas, } \\
\text { homicidios, secuestros, lesiones, agresiones, }\end{array}$ \\
\hline & \multirow[t]{2}{*}{ Corrupción } & $\begin{array}{l}\text { Tráfico de influencias, corrupción, } \\
\text { sobornos, }\end{array}$ \\
\hline & & Estafas y falsificación documentos $^{12}$ \\
\hline & Blanqueo de capitales & $\begin{array}{l}\text { Delitos fiscales } \\
\text { Blanqueo de capitales }\end{array}$ \\
\hline
\end{tabular}

Tabla 3. Distinción entre los delitos relacionados con la delincuencia organizada ${ }^{13}$

12 Algunas actividades como el blanqueo de capitales o la falsificación de documentos pueden ser actividades principales o instrumentales, según los casos.

${ }^{13}$ Debemos hacer un comentario a la hora de evaluar y analizar el cuadro presentado en la tabla n. 3. En

la actualidad, esta división tradicional entre actividades principales e instrumentales a veces no es

Revista Española de Investigación Criminológica

Artículo 9, Número 7 (2009) www.criminología.net

ISSN: $1696-9219$ 
Esta distinción es de suma importancia a la hora de evaluar la sofisticación, la complejidad y la importancia de una organización criminal. Por ejemplo, si entendemos que la amenaza de un grupo aumenta cuantos más negocios desarrolle o, lo que es lo mismo, que uno de los indicadores de riesgo de un grupo es que se dedique a más de una actividad principal, tendremos que ir con cuidado a la hora de evaluar las actividades que incluimos como actividades desarrolladas por dicho grupo. Si no procedemos a la distinción entre actividad principal e instrumental incluiríamos en las actividades del grupo todos los delitos detectados sin distinción, tendiendo a sobre-representar el riesgo de los grupos criminales detectados. De este modo, podríamos encontrar muchos más grupos que poseen actividades múltiples pero esto no significaría que sean más peligrosos, pues estaríamos calificando como actividades principales muchas que son instrumentales.

Si bien es cierta la afirmación anterior, consideramos que la clasificación entre actividad principal o instrumental no responde a una voluntad de otorgar un mayor protagonismo a la actividad principal y considerar en segundo plano las actividades instrumentales. El mayor acceso de un determinado grupo a actividades instrumentales como la violencia o el blanqueo de capitales supone, en buena lógica, que el grupo tiene una mayor capacidad, mayores recursos y, por tanto, su peligrosidad podría ser mayor. Por lo tanto, el peso de las actividades instrumentales también debe tenerse en cuenta a la hora de evaluar el riesgo de una organización, pero siendo conscientes que son actividades que no constituyen el mercado o la fuente principal de ingresos de la organización. Siendo la afirmación anterior cierta, cada vez es más común encontrar grupos de crimen organizado cuya actividad principal sea la realización de las llamadas actividades instrumentales para otros grupos. Es frecuente encontrar grupos dedicados únicamente a la falsificación de documentación, a la extorsión o al blanqueo de capitales y cuyos servicios son contratados por organizaciones externas. Debemos tomar en cuenta este aspecto ya que en estos casos las actividades instrumentales pasarían a ser principales $^{14}$.

Para proceder a medir el crimen organizado desde la clasificación mencionada con anterioridad y acudiendo a los datos policiales sobre delincuencia publicados por el Ministerio

aplicable en toda su extensión porque la tendencia de los grupos de crimen organizado es a la subcontratación de las actividades instrumentales. A pesar de ello, creemos que la distinción anterior debe servirnos de base para la valoración y evaluación de los grupos aunque matizada por la afirmación anterior.

${ }^{14}$ Asimismo, debemos también advertir de que la tendencia a la subcontratación se produce con mayor fuerza cuanto más importante sea la organización criminal. Ello nos obliga a valorar el riesgo de una organización o su potencial peligrosidad a la inversa que tradicionalmente. Los grupos menos peligrosos son los que realizan las actividades instrumentales en el seno del mismo grupo. Los grupos más grandes, por motivos de seguridad y mantenimiento de la clandestinidad, suelen subcontratar funciones como el blanqueo de capitales, la falsificación de documentación, etc. 
del Interior, nuevamente nos encontramos sin datos para proceder a ello. En la actualidad resulta inviable conocer qué tipo de delitos son cometidos por cada uno de los grupos organizados, acudiendo a la base de datos estadística sobre delincuencia. Como hemos adelantado al inicio de este articulo, de entre las categorías delictivas establecidas en dichas bases de datos no es posible desagregar los sujetos que han cometido dichos delitos con carácter individual o en el marco de un grupo de crimen organizado. Con los datos específicos de delincuencia organizada únicamente podemos conocer el porcentaje de grupos de grupos que se dedica a cada una de las actividades (ver gráfico 6). Sin embargo, esto conlleva dos problemas: que no se procede a distinguir entre actividades principales e instrumentales y que no podemos conocer la "producción delictiva de cada uno de los grupos" o número de delitos cometidos por cada uno de los grupos.

\section{Gráfico 6. Distribución de las actividades principales de delincuencia organizada $1998 / 2007^{15^{*}}$}

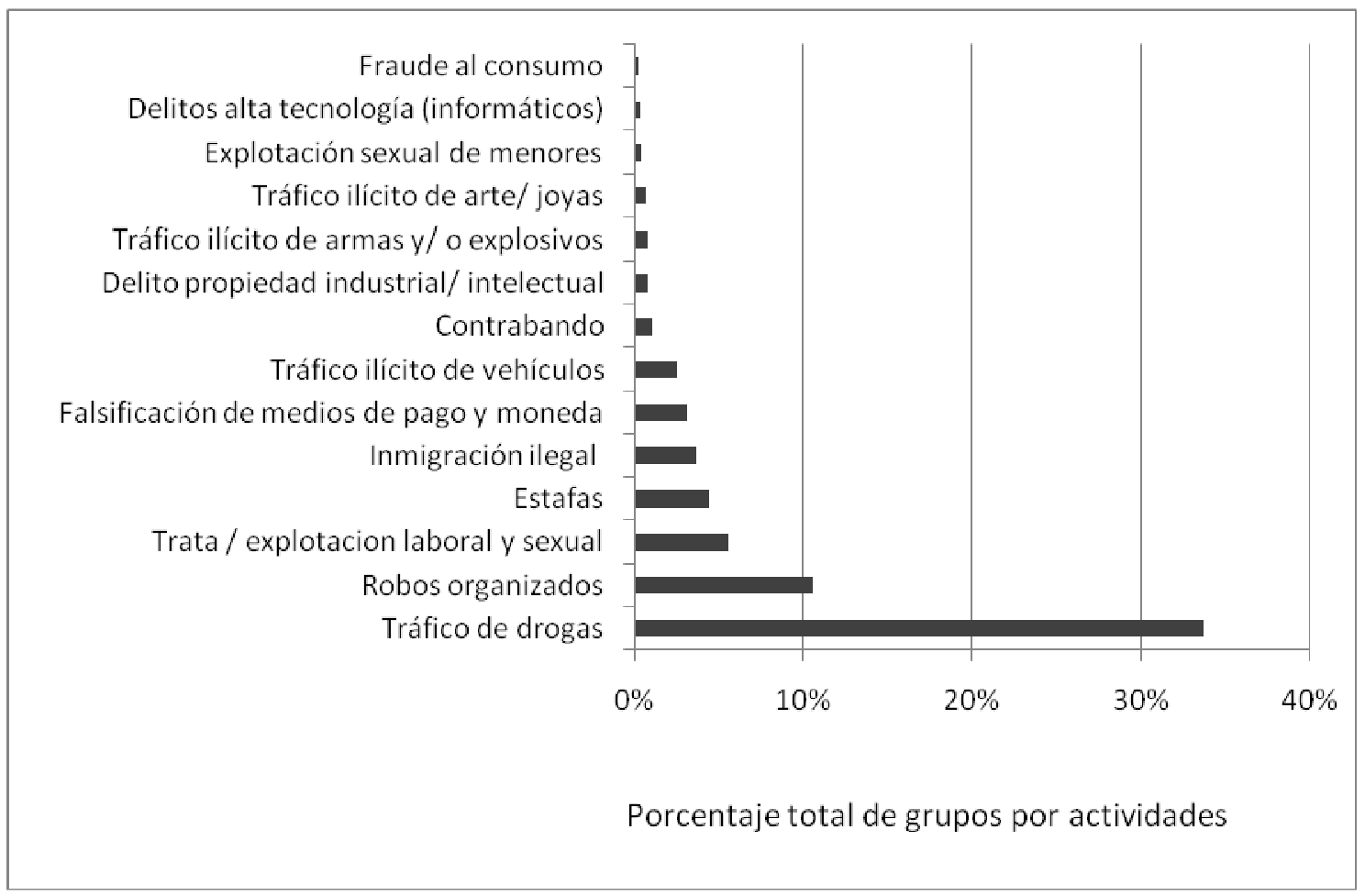

"Fuente: elaborada a partir de datos procedentes del Ministerio del Interior.

Para superar el problema que afecta a los datos oficiales como forma de medición de la delincuencia organizada, una forma muy utilizada consiste en realizar estimaciones sobre el

\footnotetext{
${ }^{15}$ El gráfico 5 representa el porcentaje medio de grupos que se dedican a las mencionadas actividades en el periodo correspondiente (1998-2007).
}

Revista Española de Investigación Criminológica 
volumen de un mercado ilegal concreto a partir de datos indirectos. En el caso del tráfico de drogas, tenemos un ejemplo en los informes que periódicamente publica Naciones Unidas sobre drogas (ver ONNUD, 2007, por ejemplo). Estas estimaciones sobre volumen de los mercados de la droga se realizan a partir de las incautaciones realizadas por las fuerzas y cuerpos de seguridad del estado. A pesar de que dicha forma de medición tenga muchas limitaciones, permite analizar tendencias y sobre todo, desplazamientos geográficos en los mercados de todo tipo de drogas. Asimismo, también se han llevado a cabo estimaciones puntuales sobre diversos tipos de mercados ilegales, como el tráfico de drogas (ONNUD, 2007), mercado de cigarrillos (Merriman, 2000), tráfico seres humanos (Di Nicola y Canduro, 2007, UNODOC, 2009), o sobre la criminalidad organizada en general (Morselli y Vannini, 1997) por poner algunos ejemplos ${ }^{16}$.

\subsection{Actividades instrumentales para el desarrollo de los mercados de criminalidad organizada}

Otra forma de abordar la medición de la delincuencia organizada consiste en evaluar y valorar las actividades que llamábamos instrumentales, necesarias para el desarrollo de los mercados de crimen organizado y que constituyen las guías o medios imprescindibles para que las organizaciones criminales conduzcan su actividad ilegal. Tres son las actividades que sabemos que constituyen las herramientas necesarias para el desarrollo del crimen organizado: el blanqueo de capitales, la violencia instrumental y la corrupción. Si procedemos a una medición de estas actividades instrumentales, podremos también acercarnos de forma indirecta a la evolución o medida del crimen organizado en un lugar determinado.

\section{Blanqueo de capitales}

Si bien es cierto que no todas las actividades de blanqueo de capitales que se detectan proceden de actividades de delincuencia organizada, su evolución puede servirnos de indicador para detectar tendencias ya que es una actividad consustancial al crimen organizado. Las organizaciones criminales necesitan de los procesos de blanqueo de capitales para poder desarrollarse, reutilizar las ganancias de procedencia ilícita y conseguir ampliar su mercado y demarcación territorial (Van Dijk, 2007).

\footnotetext{
${ }^{16}$ Existen diversos modos de acercamiento o metodologías aplicables para la estimación de mercados ilegales, especialmente a aquellos que se generan a partir de mercados legales existentes. Podemos proceder a realizar estimaciones a partir de encuestas a expertos o encuestas a consumidores. Asímismo podemos realizar estimaciones a partir de datos de importaciones o exportaciones, a partir de la comparación de datos de venta y datos de consumo de un producto ilegal o a partir de datos de consumo.
} 
Gráfico 7. Delitos detectados de blanqueo de capitales entre 1998 y 2008 por el CNP y la GC"

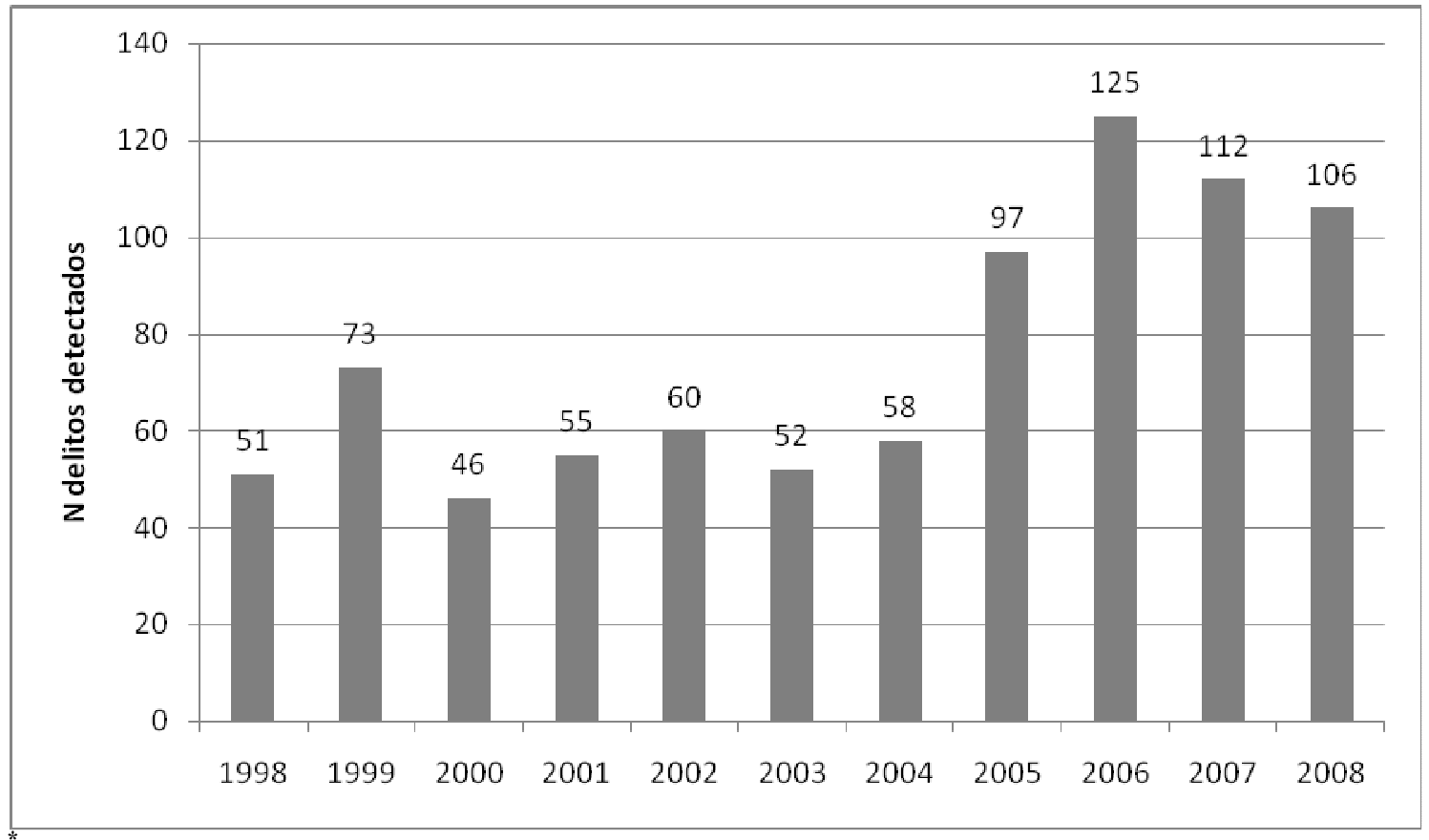

"Fuente: Elaborado a partir de los datos del Ministerio del Interior

Si analizamos en primer lugar el número de delitos de blanqueo de capitales detectados por parte de instancias policiales (grafico 7) vemos que, en los últimos diez años, ha habido una evolución ascendente hasta el año 2006 donde empieza un proceso descendente. Como puede observarse, la gráfica dibuja una evolución parecida a la evolución de la tasa de detenidos por delincuencia organizada por 100.000 habitantes que analizábamos en el gráfico 4 . 
Gráfico 8. Asuntos de blanqueo de Capitales Iniciados y Porcentaje de Variación anual de los mismos (respecto al año anterior)*

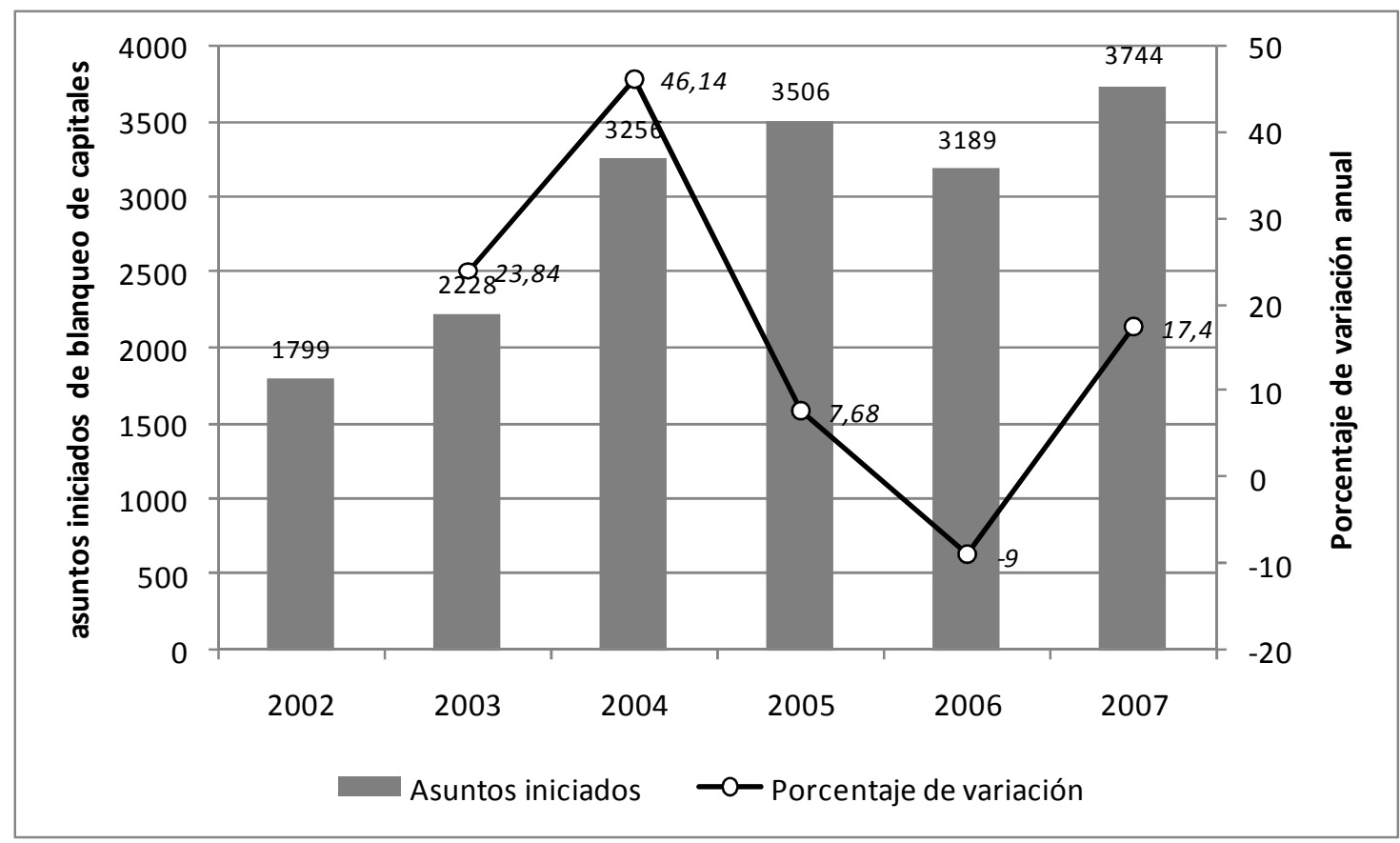

*Fuente: Memorias anuales del SEPBLAC (Servicio Ejecutivo de la Comisión de Prevención del Blanqueo de Capitales e Infracciones Monetarias. Banco de España)

Otra fuente relevante de datos sobre el blanqueo de capitales proviene del SEPBLAC (Gráfico 8). De entre los diversos datos que en estos informes figuran, son de especial interés los estadísticos referidos a asuntos iniciados por blanqueo de capitales. El gráfico 8 muestra la cantidad de asuntos iniciados desde el año 2002 a 2007 observándose un incremento de los mismos. Por otra parte, el mencionado gráfico también muestra un porcentaje de variación anual asociado a estos asuntos (línea continua). Como puede observarse desde el año 2003 al 2004, la tasa de variación es positiva mientras que la que va del año 2004 al 2006 presenta una ralentización con una posterior aceleración del 2006 al 2007. En definitiva, lo que muestra dicho gráfico es un aumento de la detección de casos de blanqueo durante los últimos años con una variación anual irregular. Así, estos datos nos ayudan a conocer tendencias sobre una actividad instrumental o transversal en el crimen organizado pero de dichos datos no podemos desagregar los referidos únicamente a casos de crimen organizado. En dichos datos pueden estar incluidos datos de blanqueo referidos a otras muchas actividades. 
Sabemos también por la literatura sobre crimen organizado (Abadinsky, 2007) que los grupos de delincuencia utilizan frecuentemente violencia física y psicológica con diferentes finalidades. La violencia es utilizada hacia el exterior de la organización por dos motivos principalmente: para evitar la competencia de otras organizaciones y conseguir el monopolio de sus actividades; y para intimidar a las autoridades públicas y desarrollar la actividad con mayor impunidad. Asimismo, la violencia es también utilizada hacia el interior de las organizaciones por otros motivos: para asegurar el mantenimiento de los códigos internos por parte de los miembros de una organización y como método de advertencia y castigo por incumplimiento de normas $\mathrm{u}$ obligaciones establecidas por la organización (De la corte y Giménez-Salinas, en prensa).

Así, si la violencia también es una actividad instrumental que se debería encontrar necesariamente en la mayoría de grupos de delincuencia organizada, una forma de medir la delincuencia organizada de un determinado país podría consistir en conocer el número de actos violentos, incluidos los homicidios, que se producen anualmente relacionados con el crimen organizado. En el caso de los homicidios, no son datos fáciles de conseguir ya que los homicidios relacionados con el crimen organizados son más complejos de investigar que otro tipo de homicidios. Así, conseguir averiguar si dichos homicidios están relacionados con el crimen organizado y en qué medida, no es tarea sencilla. Por el momento, carecemos de datos precisos sobre los homicidios ${ }^{17}$ relacionados con la delincuencia organizada así que lo que intentaremos es medir la violencia instrumental a través de otros datos sobre delitos violentos ejercidos por grupos de crimen organizado.

${ }^{17}$ A pesar de ello, la tasa de homicidios relacionados con la delincuencia organizada constituye una forma de medición especialmente indicada para establecer comparaciones con los países de nuestro entorno. 
Gráfico 9. Grupos organizados detectados que han cometido actos de extorsión, delitos contra las personas y secuestros entre 1998 y 2006*

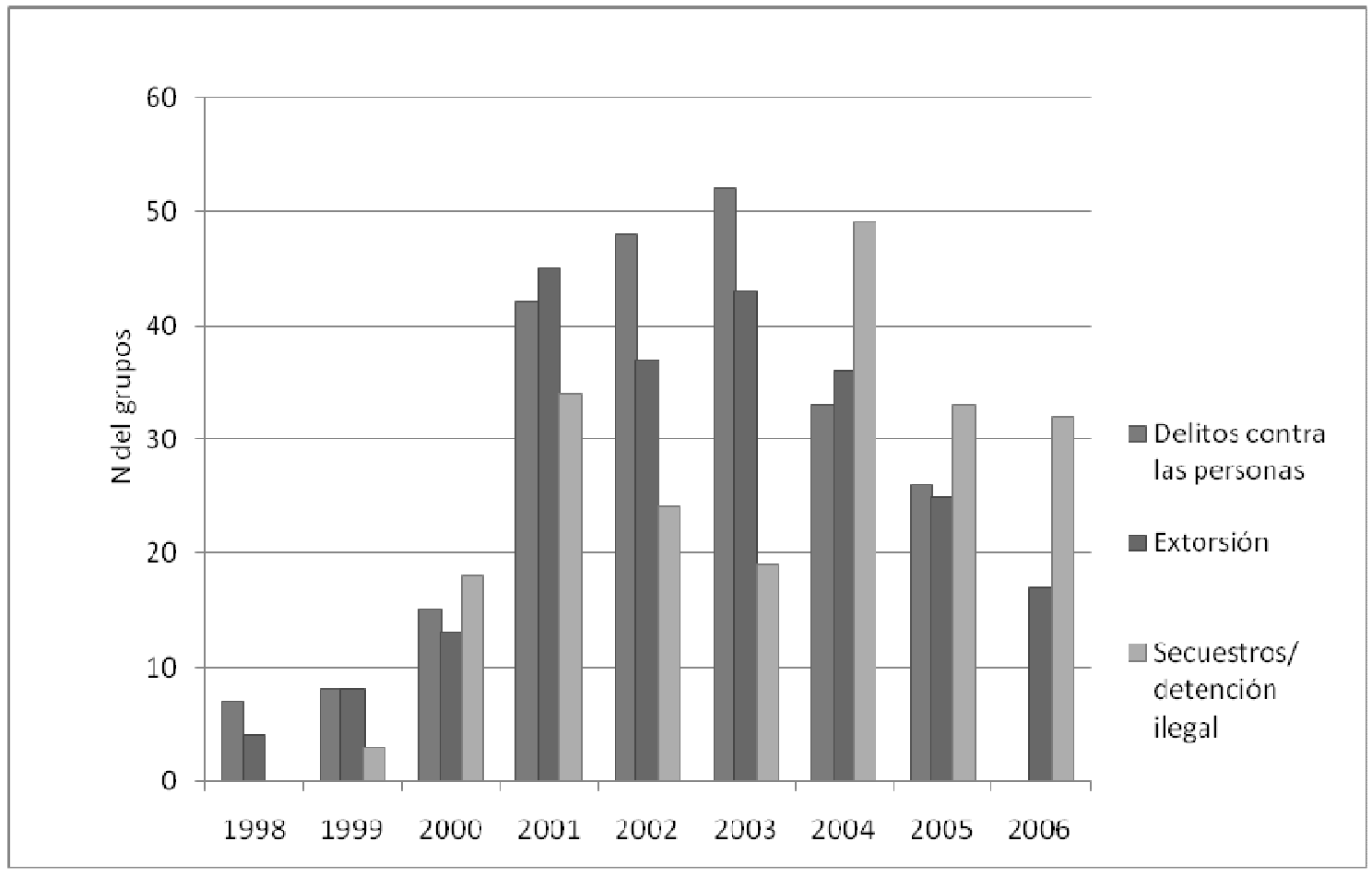

Fuente: Elaborado a partir de los datos del Ministerio del Interior

El gráfico 9 nos presenta una situación un tanto extraña que puede ser debida a la metodología de recogida de datos sobre dichas actividades en los últimos años. Sin embargo, podemos intentar interpretar las tendencias que nos muestra dicho gráfico. El gráfico 9 nos indica que, en los últimos años, después de un ascenso en el número de grupos detectados en los que se han identificado actividades propias de violencia instrumental, se esta produciendo un descenso en este tipo de actividades salvo en el caso de los secuestros. Esto podría tener, al menos, dos explicaciones. Podría explicarse por una tendencia hacia una mayor sofisticación en los métodos instrumentales utilizados por parte de los grupos de delincuencia organizada acompañada de un descenso de la violencia manifiesta. La segunda lectura iría encaminada a considerar que efectivamente ha habido un descenso de la violencia registrada por parte de los grupos y ello podría indicar que el protagonismo en la actividad de delincuencia organizada detectada lo tendrían grupos de menor envergadura y peligrosidad, que tendrían menor capacidad para ejercer formas instrumentales de violencia. Ambas interpretaciones deberían ser sometidas al refrendo empírico para poder ser contrastadas. 


\section{CONCLUSIONES}

Tal como avanzábamos en el título de este artículo, la medición del crimen organizado no es una cuestión sencilla ni disponemos en la actualidad de datos suficientes para llevarla a cabo con precisión. Los principales obstáculos encontrados se relacionan con su definición, su identidad multidimensional y la posibilidad y el acceso a los datos.

A partir de los datos policiales recogidos sobre este tipo de delincuencia, hemos conocido delincuencia organizada detectada de la última década en torno a tres dimensiones del fenómeno. En primer lugar, respecto a los grupos de delincuencia organizada, cuyos datos nos han permitido comprobar las limitaciones de dicha medida como forma de medición y evaluación del crimen organizado. Más precisa es la medición basada en el número de detenidos por delincuencia organizada a través de la que sí hemos podido comprobar una evolución ascendente de la detección de estos delitos desde 1998, existiendo solamente un descenso de 2002 a 2004.

En segundo lugar, los datos sobre actividades relacionadas con el crimen organizado únicamente nos han permitido conocer la distribución de los mercados ilegales entre los grupos detectados en los últimos diez años. Una medición y evolución mucho más elaborada debería llevarse a cabo para poder conocer con mayor profundidad no sólo las actividades delictivas de los grupos organizados sino los mercados ilegales desarrollados. En este sentido, variables ad hoc deberían crearse para medir y evaluar mejor los mercados ilegales desarrollados en España y el papel que respecto a ellos tienen los grupos detectados en nuestro territorio.

En tercer lugar, una forma indirecta de abordar la medición de la delincuencia organizada consiste en medir las actividades instrumentales que se desarrollan por parte de los grupos delictivos. Así, el blanqueo de capitales, la violencia instrumental y la corrupción son indicadores que pueden servirnos para conocer la realidad de la delincuencia organizada en un determinado lugar. Hemos podido recoger datos sobre el blanqueo de capitales y la violencia instrumental relacionada con la delincuencia organizada. Los datos sobre blanqueo de capitales nos muestran una evolución ascendente de las detecciones por parte de las autoridades policiales y administrativas en dicha materia, en consonancia con los datos sobre grupos o detenidos que se habían recogido con anterioridad. La tasa de homicidios constituye un indicador idóneo para conocer la evolución del crimen organizado y tener una referencia para la comparación internacional. Sin embargo, la recogida de datos es todavía insuficiente para llevar a cabo una medición precisa de la misma. Los datos sobre delitos violentos cometidos por los grupos de 
crimen organizado nos muestran tendencias hacia métodos instrumentales más sofisticados y menos expresivos que deberíamos comprobar.

Dada la complejidad de medida y evaluación del fenómeno de la delincuencia organizada, seria especialmente útil disponer de una metodología orientada a la evaluación de los grupos criminales que permitiera establecer tipologías y perfiles de organizaciones criminales; distinguir y definir los mercados ilícitos que se desarrollan localmente y, en definitiva, comprender las características y dinámicas del crimen organizado que se genera o actúa en nuestro país. Somos conscientes que dichas metodologías son aplicadas a nivel policial y en los departamentos de inteligencia criminal para la lucha contra el crimen organizado. Sin embargo, la investigación académica sobre este fenómeno se ve especialmente limitada por la carencia de datos y la imposibilidad de acceso a los mismos.

Finalmente, los datos policiales recogidos referentes a las tres dimensiones del crimen organizado nos muestran, aunque con diferencias, un aumento en la detección de casos de delincuencia organizada en los últimos diez años gracias al esfuerzo policial en esta materia. Sin embargo, quedaría por contrastar si este aumento representa una realidad o simplemente un aumento de la presión policial. En este sentido, sería de gran utilidad poder contar con estudios que permitieran aportar mediciones alternativas a las oficiales a través de encuestas de victimizacion a poblaciones concretas o a partir de mediciones generales tomando como referencia indicadores indirectos. Es precisamente en referencia a esta última cuestión que plantearemos nuestro trabajo en el futuro con el fin de conseguir mejorar la medición local del crimen organizado. 


\section{REFERENCIAS}

Abandinsky, H. (2007). Organized Crime. Belmont: Thomson Wadsworth.

Albanese, J. S. (2008). Risk Assessment in Organized Crime. Developing a Market and Product based Model to Determine threat Levels. Journal of Contemporary Criminal Justice, 24, 3: 263-273.

Albanese. J.S. (2001). The Prediction and Control of Organized Crime: A Risk Assessment Instrument for Targeting Law Enforcement Efforts. Trends in Organized Crime, 6, 3: 4-29.

Albanese, J. (2000). The Causes of Organized Crime: Do Criminals Organized Around Opportunities for Crime or Do Criminal Opportunities Create New Offenders? Journal of Contemporary Criminal Justice, 16: 409-423.

Beare, M (2000). Structures, Strategies and Tactics of Transnational Criminal Organizations: Critical Issues for Enforcement. Paper presented at the Transnational Crime Conference. Canberra, 9-10.

Bruinsma, G. y Bernasco, W. (2004). Criminal Groups and Transnational Illegal Markets. Crime, Law and Social Change, 41:79-94.

Cressey, D. R. (1969). The Theft of the Nation: The Structure and Operations of Organized Crime in America. New York: Harper and Row.

Edwards, A. y Levi, M. (2008). Researching the organization of serious crimes. Criminology and Criminal Justice, 8: 363-388.

Di Nicola, A. y Cauduro, A. (2007). Review of official statistics on trafficking in human beings for sexual exploitation and their validity in the $25 \mathrm{EU}$ member States: from official statistics to estimates of the phenomenon. En Savona, E, U and Stefanizzi, S. (Eds), Measuring human trafficking: complexities and pitfalls. Berlin: Springer.

Farrington, D. P. (2005). Integrated developmental and life-course theories of offending. Advances in criminological theory, 14. New Brunswick, NJ: Transaction Publishers.

Foglesong, T. (2005). Regional variation in public perceptions of organized crime in the Balkans. Centre of Organized Crime in Southeast Europe. Vera Institute for Justice.

Galeotti, M. (1998). The Mafiya and the New Russia. Australian Journal of Politics, 44, 3: 415-429.

Garrido, V., Stangeland, P. y Redondo, S. (2006). Principios de Criminología. Valencia: Tirant lo Blanch.

Jamieson, A. (2000). The Antimafia: Italy's fight against organized crime. London: Mcmillan press.

EUROPOL. European Organized Crime Threat Assessment, 2006, 2007, 2008. Disponible en: http://www.europol.europa.eu/index.asp?page=publications\&language $=$

Kleemans \& De Poot, 2008. Criminal Careers in Organized Crime and Social Opportunity Structure. European Journal of Criminology, 5, 1: 69-98.

Yurekli, A. y De Beyer, J. Understand, Measure, and Combat Tobacco Smuggling. World Bank. Economics of Tabacco Toolkit.

Levi, M. 2003. The Organization of serious crimes. En Maguire, M., Morgan, R. y Reiner, R (Eds). The Oxford handbook of Criminology. Oxford: Oxford University Press. P. 878-913.

Mcillwain, J.M. (1999). Organized crime: A social network approach. Crime, Law \& Social Change 32: 301-323.

Medina Ariza, J. J. (1999). Una introducción al estudio criminológico del crimen organizado. En Ferré Olivé, J. C. y Anali Borrallo, E. Delincuencia organizada: aspectos penales, procesales y criminológicos. Universidad de Huelva.

Merriman, David, Ayda Yurekli and Frank Chaloupka (2000). "How big is the worldwide cigarettes smuggling problem?”, 365-392. En Jha, P. y Chaloupka, F. J. Tobacco Control in Developing country. London: Oxford University Press.

Revista Española de Investigación Criminológica

Artículo 9, Número 7 (2009) www.criminología.net

ISSN: $1696-9219$ 
Morselli, C. (2009). Inside Criminal Networks. New York: Springer.

Morselli, C. (2003). Career opportunities and network-based privileges in the Cosa Nostra. Crime, Law and Social Change, 39: 383-418.

Morselli, C. y Vannini, M. (1997). Estimating a Crime Equation in the Presence of Organized Crime: Evidence from Italy. International Review of law and Economics, 17: 89-113.

ONUDD (2007). Informe mundial sobre las drogas. Naciones Unidas. Disponible en la página web: www.unodc.org.

Paoli, L. (2002). Paradoxes of organized crime. Crime, Law and Social Change, 31, 1: 51-97.

Sánchez García de Paz, I. (2005). La criminalidad organizada. Aspectos penales, procesales, administrativos y policiales. Madrid: Dyckinson.

UNODOC (2009). Global Report on Trafficking in persons. Disponible en la página web: http://www.unodc.org/documents/Global_Report_on_TIP.pdf

Van Duyne, P.C. Almir Maljevic, Van Dijk, M, Von Lampe, K, Newell, J. L. (Eds.). The Organization of Crime for Profit: Conduct, Law and Measurement. Nijmegen: Wolf Legal Publishers, 2006.

Van Dijk, J. J. M. (2008). The World of Crime. Breaking the Silence on Problems of Security, Justice and Development Across the World. Thousand Oaks: Sage.

Van Dijk, J. J. M. y Nevala, S. (2002). Intercorrelations of Crime. En Nieuwbeerta, P.: Crime victimization in international perspective. The Hague: Boom Juristische Uitgevers.

Van Dijk. J. J. M. y Buscaglia, E. (2003). Controlling organized crime and corruption in the public sector. Forum on Crime and Society, 3, 1y 2.

Van Dijk, J.J. M., y Terlouw, G. T. (1996). An International perspective of the business community as victimes of fraud and crime. Security Journal, 7, 157-167.

Vander Beken, T. (2004). Risky business: A risky based methodology to measure organized crime. Crime, Law and Social Change, 41, 5: 471-516.

Von Lampe, P. (2004). Measuring Organised Crime A Critique of Current Approaches. En Petrus C. Van Duyne, Matjaz Jager, et al. (Eds). Threats and Phantoms of Organized Crime, Corruption and Terrorism: Rhetoric and critical perspectives, p. 85-116.

Von Lampe, P. (2005). Making the Second Step before the First: Assessing Organized Crime. Crime, Law and Social Change, 42: 227-259.

Williams, P. \& Godson, R. (2002). Anticipating Organized and Transnational Crime. Crime, Law \& Social Change, 37: 311-355.

Revista Española de Investigación Criminológica

Artículo 9, Número 7 (2009) www.criminología.net

ISSN: $1696-9219$ 